\title{
Plasma instability of a vacuum arc centrifuge
}

\author{
M. J. Hole, R. S. Dallaqua,* S. W. Simpson, and E. Del Bosco* \\ School of Electrical and Information Engineering, University of Sydney, New South Wales 2006, Australia
}

(Received 30 August 2001; published 28 March 2002)

\begin{abstract}
Ever since conception of the vacuum arc centrifuge in 1980, periodic fluctuations in the ion saturation current and floating potential have been observed in Langmuir probe measurements in the rotation region of a vacuum arc centrifuge. In this work we develop a linearized theoretical model to describe a range of instabilities in the vacuum arc centrifuge plasma column, and then test the validity of the description through comparison with experiment. We conclude that the observed instability is a "universal" instability, driven by the density gradient, in a plasma with finite conductivity.
\end{abstract}

DOI: 10.1103/PhysRevE.65.046409

PACS number(s): 52.35.Kt, 52.35.Qz, 52.65.Kj, 28.60.+s

\section{INTRODUCTION}

In 1981, Krishnan, Geva, and Hirshfield [1] described a new type of plasma centrifuge, the vacuum arc centrifuge (VAC). The VAC is an axially configured plasma centrifuge, in which a uniform magnetic field is present in a cylindrical vessel. The device was initially proposed as a means to separate metal isotopes without being limited to the Alfvén critical velocity.

Figure 1(a) shows a schematic of the PCEN (plasma centrifuge) device [2] at the Brazilian National Space Research Institute (INPE), which is a typical VAC. The source of the plasma is metal ablated from the cathode, which is ionized by an electrical breakdown between the cathode and anode mesh. The discharge is triggered by either a $\mathrm{CO}_{2}$ laser $[2,3]$, which vaporizes metal from the cathode surface, or by a high-voltage trigger [4]. The VAC is usually a pulsed device, with drive currents of about $1 \mathrm{kA}$ lasting for several milliseconds. It is the interaction of the radial current and the axial magnetic field that sets the plasma into rotation, typically at frequencies between 30 and $400 \mathrm{krad} \mathrm{s}^{-1}$ [5-7]. The configuration of the anode mesh determines the radial electric field profile at that location, and has a large effect on the behavior of the plasma downstream in the rotation region [7]. The rotating plasma column streams supersonically through the anode mesh, and is kept in confinement with the balance of centrifugal, Lorentz $\mathbf{J} \times \mathbf{B}$ forces and the pressure gradient. Centrifuging leads to isotopic separation, which can be measured by sampling with a mass spectrometer during the discharge [8] or afterwards in the metal film deposited on the end plate [9].

In 1981, Geva, Krishnan, and Hirshfield [10] presented a set of Langmuir probe measurements for a carbon plasma. At a given axial location, two probes were placed $90^{\circ}$ apart in azimuth. The floating potential and ion density signals from both probes exhibited the same characteristic fluctuation frequency, with a phase difference of a quarter of a period. Measurements of the ion saturation current at different radii revealed that the fluctuation frequency was independent of

\footnotetext{
*Permanent address: Laboratório Associado de Plasma (LAP), Instituto Nacional de Pesquisas Espaciais (INPE/MCT), CP 515, 12201-970, São José dos Campos, SP, Brazil.
}

radius within the bulk of the Gaussian density distribution. Measurements of the ion saturation current at locations $0.3 \mathrm{~m}$ apart in the axial direction showed that the fluctuation occurred at the same frequency and with no phase difference. Similar results were quoted for $\mathrm{Al}-\mathrm{Ti}$ and $\mathrm{Cu}-\mathrm{Ni}$ plasmas, albeit at slightly lower frequencies, implying that any axial wavelength is significantly greater than $0.3 \mathrm{~m}$.

Since that time, numerous other measurements of the azimuthal structure of the oscillation have been made, all of which suggest the presence of a rotating nonuniformity with azimuthal mode structure $m=1[3,4,6-8,11,12]$. Reversal of the magnetic field has been shown to reverse the direction of

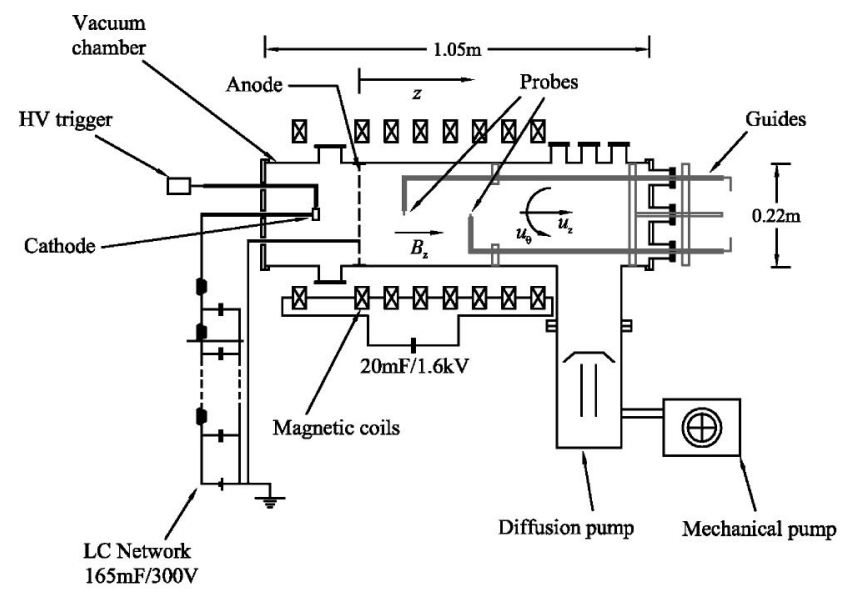

(a)

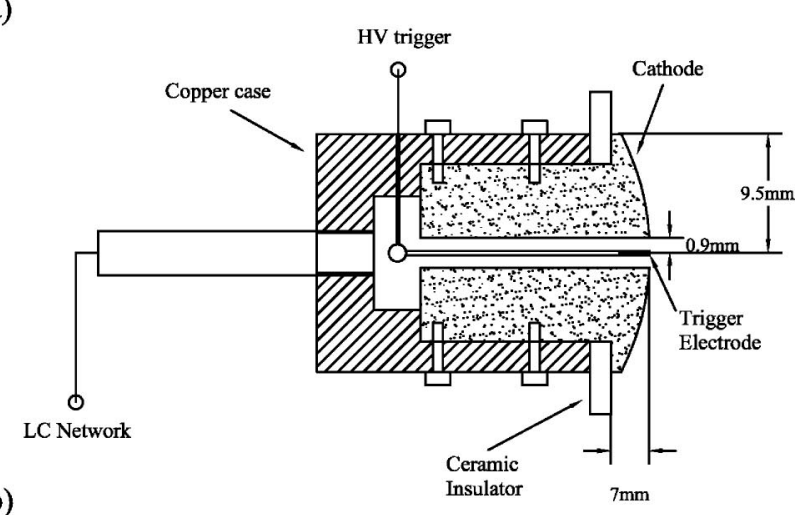

FIG. 1. (a) Schematic of the PCEN device and (b) schematic of the magnesium cathode. 
plasma rotation in both experiment [3,11] and theory [7], and the direction of nonuniformity rotation in the experiment [3]. The nonuniformity rotates in the same sense as the plasma [3]. In addition, the nonuniformity vanished in the presence of a substantial amount of background gas [12], and a fluid simulation of the VAC indicated that the driving mechanism for the nonuniformity may be associated with the effects of electron-ion collisions perpendicular to the magnetic field [13].

Several experiments suggest that the measured oscillation frequency is close to the plasma rotation frequency, but the data is not conclusive $[2,3,5,14]$. In the Yale University VAC device with a carbon cathode, Geva, Krishnan, and Hirshfield [3] report a Langmuir probe frequency of $200 \mathrm{krad} \mathrm{s}^{-1}$ for $B_{z}=0.13 \mathrm{~T}$, whilst Prasad and Krishnan [5] report that the spectroscopically inferred ion rotation frequency is linear with the magnetic field across the range $0.07<B_{z}<0.21 \mathrm{~T}$ for a discharge current of $2.1 \mathrm{kA}$. For $B_{z}=0.13 \mathrm{~T}$, their fit of angular rotation frequency to magnetic field variation infers an ion angular rotation frequency of $120 \mathrm{krad} \mathrm{s}^{-1}$, which is somewhat lower than the probe frequency at $200 \mathrm{krad} \mathrm{s}^{-1}$. In the PCEN device with a magnesium cathode, Dallaqua, Simpson, and Del Bosco [14] report a probe frequency of $200 \mathrm{krad} \mathrm{s}^{-1}$ for $B_{z}=0.15 \mathrm{~T}$. For identical conditions, Dallaqua et al. [2] estimated the axial streaming velocity of the plasma, inferred from a measurement of the time displacement of two axially separated Langmuir probes. Combining this estimate with the angle of rotation of the shadow of a thin ceramic rod obstructing the upstream plasma, they inferred an ion rotation frequency of $170 \mathrm{krad} \mathrm{s}^{-1}$, which is also somewhat lower than the probe frequency at 200 $\mathrm{krad} \mathrm{s}^{-1}$. Whilst detailed measurements of spectroscopic and probe frequencies under identical conditions are clearly warranted, these experiments indicate that the nonuniformity frequency is either comparable to or slightly greater than the ion rotation frequency.

In summary, experimental evidence to date suggests an $m=1$ nonuniformity $[3,4,6-8,10-12]$ that rotates in the same sense as the plasma [3], with angular frequency either comparable to or slightly above the plasma angular rotation frequency $[2,3,5,14]$. The nonuniformity is present over a wide range of plasma compositions, but is suppressed when a substantial amount of background gas is present [12]. Finally, the axial wavelength for $\mathrm{C}, \mathrm{Al}-\mathrm{Ti}$, and $\mathrm{Cu}-\mathrm{Ni}$ plasmas is significantly longer than $0.3 \mathrm{~m}$ [10].

Instabilities have been observed in other laboratory rotating plasmas, including $Q$ machines [15], $\theta$ pinches [16,17], and magnetic mirrors [18-20]. Of these, the low- $\beta Q$ machines with no curvature of the magnetic field are most similar to the VAC; the $Q$ machine instability research has been summarized by Motley [15]. Despite the similarities, there are still several major differences between $Q$ machine and VAC plasmas. In contrast to the VAC plasma, the $Q$ plasma is created by contact ionization of atoms and thermionic emission of electrons at the hot end plates and is at most singly ionized. In the VAC plasma, rotation is set up by current flow in the drive region [7] leading to $\partial \phi(r) / \partial r$ $>0, \partial p(r) / \partial r<0$ in the downstream rotation region, where $\phi$ is the electric potential, $p$ is the total pressure, and $r$ is the radius. In a $Q$ machine, the radial electric field is determined by the balance of electron fluxes into and out of the end plates [15]; for the ideal $Q$ machine the end plates are uniformly heated and so $\partial \phi(r) / \partial r<0, \partial p(r) / \partial r<0$. In the laboratory frame, the VAC plasma is transported supersonically along the magnetic field, whereas the $Q$ machine plasma is stationary. The VAC plasma is isolated from the conducting walls, whereas the $Q$ plasma is aperture limited in the radial direction [21]. Finally, the $Q$ machine plasma is in electrical contact with both end plates. In contrast, the VAC axial boundary condition is that the plasma should take the potential profile of the anode mesh. The VAC collector end-plate conditions vary with the experiment, ranging from the conducting vacuum vessel wall to mylar film.

The following section describes the assumptions made, introduces the plasma model, and solves for the steady state. Section III introduces the wave perturbation, reduces the system of equations to a five-row matrix, and discusses perturbation boundary conditions. Sections IV and V, respectively, solve the system of equations with the absence and inclusion of electron-ion collisions; for each case dispersion relations are derived and unstable modes investigated. In Sec. VI, experimental measurements in the PCEN device are described and in Sec. VII experimental results are compared to theory. Finally, Sec. VIII contains concluding remarks.

\section{ASSUMPTIONS, PLASMA MODEL, AND STEADY-STATE SOLUTION}

\section{A. Assumptions}

The plasma is described by a two-fluid model in a cylindrical coordinate system $(r, \theta, z)$. Following earlier models of the VAC plasma [22,23], the following assumptions are made about the plasma in the rotation region.

(1) Ions of different charge can be treated as a single species with average charge $Z$.

(2) Quasineutrality, such that $n_{e}=Z n_{i}$.

(3) A steady-state plasma, which is azimuthally symmetric and has no axial structure.

(4) Steady-state ion and electron species velocities, $\mathbf{v}_{i}$ $=\left(0, \omega_{i} r, v_{i z}\right)$ and $\mathbf{v}_{e}=\left(0, \omega_{e}(r) r, v_{e z}(r)\right)$ where $\omega_{i}$ is the ion rigid-rotor rotation frequency, $v_{i z}$ is the ion uniform axial streaming velocity, $\omega_{e}(r)$ is the electron rotation frequency, and $v_{e z}(r)$ is the electron streaming velocity. Ion rigid rotation has been established from spectroscopic measurements [24], whilst radially uniform ion streaming velocity has been established from plasma deposition experiments $[2,4,14]$. Radial diffusion of both ion and electron species due to electron-ion collisions is small $[13,25]$ and is neglected in this treatment. The neglect of classical diffusion in this work is consistent with the treatment by Chen [26] in linear geometry, for which there was no zero-order electric field. Whilst the rotating plasma treatment by Chen [27] included classical diffusion in the steady state, it was not considered in the ensuing wave treatment.

(5) Uniform ion and electron temperature, $T_{i}$ and $T_{e}$. Spectroscopic measurements of ion temperature from line broadening indicate that the ion temperature is uniform across the column [24]. A comparison with experimental data 
from the VAC indicates that the ion and electron temperature are similar within the plasma column $[12,24]$.

(6) Low magnetic Reynold's number [25]. Plasma induced fluctuations in the externally applied magnetic field are neglected. The electric field is thus $\mathbf{E}=-\boldsymbol{\nabla} \phi$.

(7) Steady-state ion density distribution $n_{i}$ $=n_{i}(0) e^{-(r / R)^{2 q}}$, where $n_{i}(0)$ is the on-axis ion density, $R$ is the characteristic radius (the radius at which the density is $1 / e$ of its on-axis value), and $q$ is a positive integer. For typical VAC conditions, measurements of the ion saturation current indicate $q=1[3,4,6]$, a Gaussian profile.

(8) Neglect of finite Larmor radius (FLR) and viscosity effects. With no radial diffusion, rigid ion rotation, and uniform ion streaming velocity, ion viscosity terms vanish from the steady state. Except for magnesium plasmas, estimates [28] of the ion Larmor radius are significantly less than the characteristic radius over a wide range of VAC plasmas.

(9) Neglect of electron inertia, which is reasonable for the range of frequencies considered in this work.

\section{B. Plasma model}

In this treatment we normalize length to $R$ and time to $1 / \omega_{i c}$, where $\omega_{i c}=Z e B_{z} / m_{i}$ is the ion cyclotron frequency. With these selections the coordinate system is $\tau=\omega_{i c} t$ and $(x, \theta, z)=(r / R, \theta, z / R)$, where $x$ and $z$ are the normalized radius and axial position. The equations of motion and continuity of the ion and electron fluids can be written as

$$
\begin{aligned}
\frac{\partial \mathbf{u}_{i}}{\partial \tau}+\left(\mathbf{u}_{i} \cdot \boldsymbol{\nabla}\right) \mathbf{u}_{i}= & -\psi\left(Z \boldsymbol{\nabla} \chi+\lambda \boldsymbol{\nabla} l_{i}\right)+\mathbf{u}_{i} \times \hat{\boldsymbol{z}} \\
& +\delta n_{s} \widetilde{\boldsymbol{\xi}} \cdot\left(\mathbf{u}_{e}-\mathbf{u}_{i}\right), \\
\psi Z\left(-\boldsymbol{\nabla} l_{i}+\boldsymbol{\nabla} \chi\right)- & \mathbf{u}_{e} \times \hat{\boldsymbol{z}}+\delta n_{z} \widetilde{\boldsymbol{\xi}} \cdot\left(\mathbf{u}_{i}-\mathbf{u}_{e}\right)=0, \\
-\frac{\partial l_{i}}{\partial \tau}= & \boldsymbol{\nabla} \cdot \mathbf{u}_{i}+\mathbf{u}_{i} \cdot \boldsymbol{\nabla} l_{i}, \\
- & \frac{\partial l_{i}}{\partial \tau}=\boldsymbol{\nabla} \cdot \mathbf{u}_{e}+\mathbf{u}_{e} \cdot \boldsymbol{\nabla} l_{i}
\end{aligned}
$$

with the terms defined as follows:

$$
\begin{gathered}
\mathbf{u}_{i}=\frac{\mathbf{v}_{i}}{\omega_{i c} R}=\left(x \varphi_{i}, x \Omega_{i}, u_{i_{*}}\right), \quad \mathbf{u}_{e}=\frac{\mathbf{v}_{c}}{\omega_{i c} R}=\left(x \varphi_{e}, x \Omega_{e}, u_{e^{*}}\right), \\
\lambda=\frac{T_{i}}{T_{e}}, \quad \psi=\frac{k_{B} T_{e}}{M \omega_{i e}^{2} R^{2}}, \\
\chi=\frac{e \phi}{k_{B} T_{e}}, \quad \widetilde{\boldsymbol{\xi}}=\operatorname{diag}\left(\xi_{\perp}, \xi_{\perp}, 1\right), \\
l_{i}=\ln \frac{n_{i}}{n_{i}(0)}, \quad n_{s}=\frac{\nu_{e i}}{\nu_{e i}(0)}, \quad \delta=\frac{e Z n_{i 0}}{B_{z}} \frac{\eta_{L}}{\gamma_{E}} .
\end{gathered}
$$

Here $\varphi_{i}$ and $\varphi_{e}$ are the normalized ion and electron radial velocities, respectively, divided by $x$. The terms $\Omega_{i}$ and $\Omega_{e}$ are the normalized ion and electron rotation frequencies, and $u_{i_{\varkappa}}$ and $u_{e_{\Sigma}}$ are the normalized ion and electron axial velocities. The term $\lambda$ is the ratio of the ion to electron temperature, $\psi$ is a convenient constant which for $\lambda=1$ becomes the square of the normalized ion thermal velocity, $\chi$ is a normalized electric potential $\phi, l_{i}$ is the logarithm of the ratio of the ion density $n_{i}$ to its on-axis value $n_{i}(0)$, and $n_{s}$ is the ratio of the electron-ion collision frequency $\nu_{e i}$ to its on-axis value $\nu_{e i}(0)$.

The term $\delta$ in Eqs. (1) and (2) is the normalized resistivity parallel to the magnetic field, where $\eta_{L}$ is the electrical resistivity of a Lorentz gas and $\gamma_{E}$ is the ratio of the conductivity of a charge state $Z$ to that in a Lorentz gas [29]. Within the bulk of the plasma column the Debye logarithm $\ln \Lambda$ is relatively insensitive to variations in $n_{e}$, and so evaluation on-axis is used. In addition, $\ln \Lambda$ is corrected by 0.3 to account for shielding by positive ions [30]. The dependence of $\delta$ on the electron-ion collision frequency $\nu_{\mathrm{ei}}$ and the electron cyclotron frequency $\omega_{\mathrm{ec}}$ can be found by writing $\eta_{L} / \gamma_{E}$ $\simeq m_{e} \nu_{e i} / n_{e} e^{2}$ [31], from which it is found that

$$
\delta \simeq \frac{\overline{\nu_{e i}(0)}}{\omega_{e_{z}}}
$$

which is the inverse of the electron Hall parameter evaluated on-axis. For typical VAC conditions, the parameter $\delta$ is of the order of $10^{-2}$ [28]. Finally, $\widetilde{\boldsymbol{\xi}}$ is a diagonal tensor, where $\xi_{\perp}$ is an enhancement factor of resistivity transverse to the magnetic field. The factor $\xi_{\perp}$ is equivalent to the factor $f$ in the fluid treatment of Yue and Simpson [13], which accounted for nonuniform properties and instabilities that may exist in the plasma.

\section{Steady-state solution}

In this work, the subscript " 0 " refers to the steady-state solution. Under the steady-state assumptions (3), (4), and (7) we find $\varphi_{i 0}=\varphi_{e 0}=0, l_{i 0}=-x^{2 q}$, and $n_{s 0}=\exp \left(-x^{2 q}\right)$. Integrating the radial component of Eq. (1) with respect to $x$ yields the normalized potential

$$
\chi_{0}(x)=\chi_{c}+\frac{\Omega_{i 0}}{2 \psi Z}\left(1+\Omega_{i 0}\right) x^{2}+\frac{\lambda}{Z} x^{2 q},
$$

where $\chi_{c}$ is an arbitrary reference potential. Solving the radial component of Eq. (2) for $\Omega_{e 0}$ yields

$$
\Omega_{e 0}(x)=\Omega_{i 0}\left(1+\Omega_{i 0}\right)+2 \psi(\lambda+Z) q x^{2(q-1)},
$$

which for $q=1$ is a constant. Finally, solving the axial component of Eq. (2) yields $u_{e_{\star} 0}(x)=u_{\alpha 0}$.

\section{PERTURBATION TREATMENT}

We consider the presence of waves of the form $\exp [i(m \theta$ $\left.\left.+k_{z} z-\omega \tau\right)\right]$, where $m$ is the azimuthal mode number, $k_{z}$ is the axial component of the wave vector, and $\omega=\omega^{r}+i \omega^{i}$. We restrict $\omega^{r} \geqslant 0$ such that the signs of $m$ and $k_{\Sigma}$ determine the direction of wave propagation in the $\theta$ and 2 component directions. The aim is to find dispersion relations $\omega$ 
$=\omega_{M}\left(m, k_{2}\right)$, where the subscript $M$ denotes an arbitrary wave mode.

The system of equations (1)-(4) is linearized with perturbations of the form

$$
\zeta(\tau, x, \theta, 2)=\zeta_{0}(x)+\varepsilon \zeta_{1}(x) \exp i\left(m \theta+k_{2}-\omega \tau\right),
$$

where $\zeta(\tau, x, \theta, z)$ is a place holder representing the velocity components, the logarithm of the ion density $l_{i}$, and the normalized potential $\chi$. The logarithm of the ion density $l_{i}$ can be written as

$$
l_{i}(\tau, x, \theta, z)=-x^{2 q}+\varepsilon l_{i 1}(x) \exp i\left(m \theta+k_{z^{z}}-\omega \tau\right) .
$$

The VAC plasma column is isolated from the conducting walls and surrounded by a vacuum. At large radial distances we require that the perturbation in the ion density vanish. In the limit that the conducting wall at $x=X$ is removed to infinity, the radial boundary condition can be written as

$$
\lim _{X \rightarrow \infty} l_{i 1}(X)=0 \text {. }
$$

For the modes considered in this treatment it can be shown that the relationship between $l_{i 1}(x)$ and $\chi_{1}(x)$ is linear [28]. Thus, the boundary condition $l_{i 1}(X)=0$ is equivalent to $\chi_{1}(X)=0$.

The expression for $n_{s}$, the normalized electron-ion collision frequency, becomes

$$
n_{s}=n_{s 0}(x)+\varepsilon n_{s 1}(x) \exp i\left(m \theta+k_{2}-\omega \tau\right)+O\left(\varepsilon^{2}\right),
$$

where

$$
\begin{gathered}
n_{s 0}(x)=\exp \left(-x^{2 q}\right), \\
n_{s 1}(x)=l_{i 1}(x) \exp \left(-x^{2 q}\right) .
\end{gathered}
$$

In the axial direction, the VAC boundary condition is that the plasma takes the potential profile of the anode mesh, which will short circuit any potential oscillations. This condition disallows excitation of $k_{z}=0$ or "flute" modes. Nevertheless, flute modes are investigated in this treatment for the following reasons: (a) the derivation clarifies existing knowledge [27,33], (b) the flute mode result is generalized for arbitrary $q$, and (c) analytical solutions are available, which provide a convenient check of convergence of the numerical solution presented in Sec. V.

The remaining perturbed parameters are $\mathbf{u}_{i}, \mathbf{u}_{e}$, and $\chi$, written as $\quad \mathbf{u}_{i 1}(x)=\left(x \varphi_{i 1}(x), x \Omega_{i 1}(x), u_{i \gtrless 1}(x)\right), \quad \mathbf{u}_{e 1}(x)$ $=\left(x \varphi_{e 1}(x), x \Omega_{e 1}(x), u_{e: 1}(x)\right)$, and $\chi_{1}(x)$. The plasma parameters $\zeta(\tau, x, \theta, 2)$ must be continuously differentiable along a straight line through the center of the plasma column. This property requires that the perturbations $\varphi_{i 1}(x), \Omega_{i 1}(x)$, $u_{i_{*} 1}(x), \varphi_{e 1}(x), \Omega_{e 1}(x), u_{e \gtrless 1}(x), l_{i 1}(x)$, and $\chi_{1}(x)$ all take the parity of $m$ [28]. The system comprising Eqs. (1)-(4) can now be solved.

Taking the difference between azimuthal components of the ion and electron equations, Eqs. (1) and (2), respectively, and solving for $\Omega_{i 1}(x)$ yields

$$
\Omega_{i 1}(x)=\frac{1}{\varpi}\left(m \psi(\lambda+Z) \frac{l_{i 1}(x)}{x^{2}}+i\left[\varphi_{e 1}(x)-C \varphi_{i 1}(x)\right]\right),
$$

where $\varpi=\omega-m \Omega_{i 0}-k_{z} u_{z_{0} 0}$ is the frequency in the frame of the ion fluid and $C=1+2 \Omega_{i 0}$, following the notation introduced by Chen [27]. In the typical VAC setup the target is biased negatively with respect to the mesh and so $E_{r}<0$ in the rotation region plasma [7], resulting in $\Omega_{0}>0$ and hence $C>1$.

Solving the difference between axial components of Eqs. (1) and (2) for $u_{i_{\star} 1}(x)$ yields

$$
u_{i \neq 1}(x)=\frac{k_{z} \psi(\lambda+Z)}{\omega_{s}} l_{i 1}(x),
$$

while solving the radial component of Eq. (2) for $\varphi_{e 1}(x)$ yields

$$
\begin{aligned}
\Omega_{\mathrm{el}}(x)= & \psi Z\left(-\frac{l_{i 1}^{\prime}(x)}{x}+\frac{\chi_{1}^{\prime}(x)}{x}\right)+\delta \xi_{\perp} e^{-x^{2}}\left[\varphi_{i 1}(x)\right. \\
& \left.-\varphi_{e l}(x)\right]
\end{aligned}
$$

Using the expressions for $\Omega_{i 1}, \Omega_{e 1}$, and $u_{i ₹ 1}$ together with the assumed form of $n_{s 1}$, given by Eq. (11), Eqs. (1)-(4) can be expanded to $O(\delta)$ as follows:

$$
\begin{aligned}
& \varphi\left(\lambda l_{i 1}^{\prime}(x)+Z \chi_{1}^{\prime}(x)\right)=\frac{m \psi(\lambda+Z) C}{\varpi} \frac{l_{i 1}(x)}{x} \\
& +\left(i \varpi-\frac{i C^{2}}{\varpi}-\delta \xi_{\perp} e^{-x^{2}}\right) x \varphi_{i 1}(x) \\
& +\left(\frac{i C}{\varpi}+\delta \xi_{\perp} e^{-x^{2}}\right) x \varphi_{e 1}(x), \\
& x \varphi_{i 1}^{\prime}(x)=i\left[\varpi-\frac{\psi(\lambda+Z)}{\varpi}\left(\frac{m^{2}}{x^{2}}+k_{z}^{2}\right)\right] l_{i 1}(x) \\
& -\left(2-2 q x^{2 q}+\frac{m C}{\varpi}\right) \varphi_{i 1}(x)+\frac{m}{\varpi} \varphi_{e 1}(x), \\
& x \varphi_{e 1}^{\prime}(x)+i m \psi Z\left(-\frac{l_{i 1}^{\prime}(x)}{x}+\frac{\chi_{1}^{\prime}(x)}{x}\right) \\
& =i\left\{\varpi-m\left[\Omega_{i 0}^{2}+2 \psi(\lambda+Z) q x^{2(q-1)}\right]\right\} l_{i 1}(x) \\
& -i m \delta \xi_{\perp} e^{-x^{2}} \varphi_{i 1}(x)-\left(2-2 q x^{2 q}-i m \delta \xi_{\perp} e^{-x^{2}}\right) \\
& \times \varphi_{e 1}(x)-i k_{z} u_{3 ₹ 1}(x) \text {, } \\
& \psi Z \delta \xi_{\perp} e^{-x^{2}}\left[l_{i 1}^{\prime}(x)-\chi_{1}^{\prime}(x)\right] \\
& =i m \psi Z\left(\frac{l_{i 1}(x)}{x}-\frac{\chi_{1}(x)}{x}\right)-\delta \xi_{\perp} e^{-x^{2}}[\psi(\lambda+Z) \\
& \left.\times\left(\frac{m}{\varpi}-2 q x^{2 q}\right)-x^{2} \Omega_{i 0}^{2}\right] \frac{l_{i 1}(x)}{x}+\frac{i C \delta \xi_{\perp} e^{-x^{2}}}{\varpi} \\
& \times x \varphi_{i 1}(x)-\left(1+\frac{\delta \xi_{\perp} e^{-x^{2}}}{\varpi}\right) x \varphi_{e 1}(x),
\end{aligned}
$$




$$
\begin{aligned}
i \psi Z k_{*}\left[l_{i 1}(x)-\chi_{1}(x)\right]= & \delta e^{-x^{2}}\left(\frac{k_{z} \psi(\lambda+Z)}{\omega_{s}} l_{i 1}(x)\right. \\
& \left.-u_{e_{*} 1}(x)\right)
\end{aligned}
$$

Introducing the new constant $\Psi=(\lambda+Z) \psi$, new independent variable $y=x^{2}$, and new dependent variable $X_{1}(y)$, where $X_{1}(y)=(Z / \lambda+Z)\left[l_{i 1}(y)-\chi_{1}(y)\right]$, and replacing $\psi$ and $\chi_{1}$, Eqs. (15)-(19) can be rewritten as

$$
\left(\begin{array}{c}
\psi\left(l_{i 1}^{\prime}(y)-X_{1}^{\prime}(y)\right) \\
y \varphi_{i 1}^{\prime}(y) \\
y \varphi_{e 1}^{\prime}(y)-i m \Psi X_{1}^{\prime}(y) \\
\Psi \delta \xi_{\perp} e^{-y^{e}} X_{1}^{\prime}(y) \\
0
\end{array}\right)=\overline{\mathbf{A}}\left(\begin{array}{c}
l_{i 1}(y) \\
X_{1}(y) \\
\varphi_{i 1}(y) \\
\varphi_{e 1}(y) \\
u_{e x 1}(y)
\end{array}\right)
$$

where $\tilde{\mathbf{A}}$ is the matrix

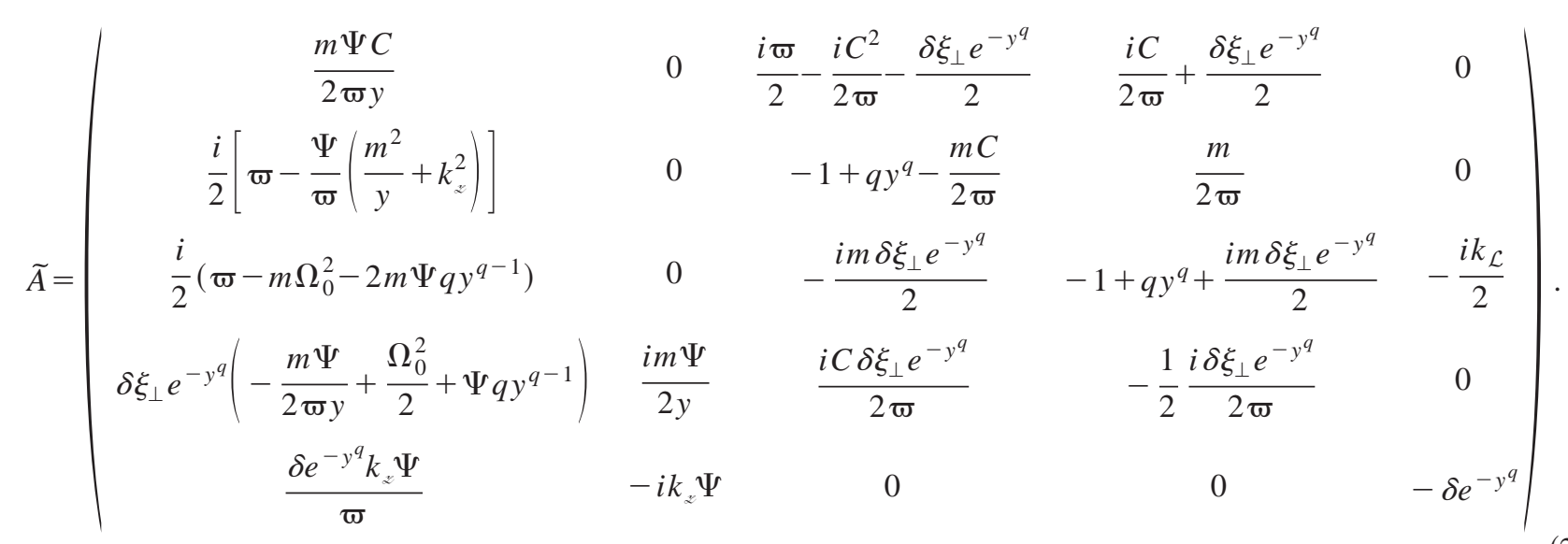

\section{PERTURBATION SOLUTIONS WITHOUT ELECTRON-ION COLLISIONS}

For the case without collisions, $\delta=0$, the fourth row of Eq. (20) gives

$$
\varphi_{e 1}(y)=i m \Psi \frac{X_{1}(y)}{y}
$$

and Eq. (20) becomes

$$
\left(\begin{array}{c}
\Psi\left(l_{i 1}^{\prime}(y)-X_{1}^{\prime}(y)\right) \\
y \varphi_{i 1}^{\prime}(y) \\
0 \\
0
\end{array}\right)=\widetilde{\mathbf{A}}_{0}\left(\begin{array}{c}
l_{i 1}(y) \\
X_{1}(y) \\
\varphi_{i 1}(y) \\
u_{e \leftleftarrows 1}(y)
\end{array}\right)
$$

where $\widetilde{\mathbf{A}}_{0}$ is the reduced matrix

$$
\widetilde{\mathbf{A}}_{0}=\left(\begin{array}{cccc}
\frac{i n \Psi C}{2 \varpi y} & -\frac{m \Psi C}{2 \varpi y} & \frac{i \varpi}{2}-\frac{i C^{2}}{2 \varpi} & 0 \\
\frac{i}{2}\left[\varpi-\frac{\Psi}{\varpi}\left(\frac{m^{2}}{y}+k_{z}^{2}\right)\right] & \frac{i m^{2} \Psi}{2 \varpi y} & -1+q y^{q}-\frac{m C}{2 \varpi} & 0 \\
i\left[\frac{\varpi}{2}-m\left(\frac{m \Omega_{0}^{2}}{2}+\Psi\right)\right] & i m \Psi q y^{q-1} & 0 & -\frac{i k_{z}}{2} \\
0 & -i k_{z} \Psi & 0 & 0
\end{array}\right) .
$$


The fourth-row equation in Eq. (23) decouples and has three solutions: (a) $k_{z}=0$, flute modes; (b) $X_{1}(y)=0$, other modes; and (c) $\Psi=0$, a cold plasma. For (c), Eq. (23) gives neutrally stable solutions that are not considered further.

\section{A. Flute modes}

For flute modes with $m=0$, the third-row equation of Eq. (23) yields $\varpi=\omega=0$ such that the perturbation defined by Eq. (7) is no longer a wave. Thus, attention is restricted to a warm plasma with $m \neq 0$.

An expression for $\varphi_{i 1}(y)$ is found from the first row of Eq. (23). Next, $X_{1}(y)$ is replaced by the new dependent variable $g_{1}(y)=X_{1}(y)-l_{i 1}(y)$, and the third row of Eq. (23) is solved for $l_{i 1}(y)$, yielding

$$
l_{i 1}(y)=\frac{-2 m q \Psi y^{q-1} g_{1}(y)}{\varpi-m \Omega_{0}^{2}} .
$$

Finally, expressions for $\varphi_{i 1}(y), X_{1}(y)$, and $l_{i 1}(y)$ are substituted into the second row of Eq. (23), giving the secondorder differential equation

$$
\left(\frac{\varpi^{2}-C^{2}}{\varpi}\right) L\left(N_{F}\right)\left[g_{1}(y)\right]=0,
$$

where $L(N)$ is the differential operator

$$
L(N)=y \frac{\partial^{2}}{\partial y^{2}}+\left(1-q y^{q}\right) \frac{\partial}{\partial y}+\left(\frac{N}{2}-\frac{m^{2}}{4 y}\right),
$$

the term $N_{F}=N_{a} y^{q-1}$, and

$$
N_{a}=\frac{m\left(\varpi^{2}-C^{2}\right)}{\left(\varpi-m \Omega_{0}^{2}\right)}+\frac{m C}{\varpi} .
$$

The stable zeros of Eq. (26) at $\varpi= \pm C$ are not considered further. For $q=1$, the $L(m, N)$ operator can be transformed to the Kummer or Whittaker equation [32]. In the limit of small rotation and small growth such that $\varpi^{3} \ll m C \Omega_{0}^{2}$, we recover the expression for $N_{a}$ derived by Chen [27] in the absence of FLR effects [in that work, Eq. (26) with $\left.r_{0}^{-2} \rightarrow 0\right]$.

The solution for $g_{1}(y)$ is [32]

$$
\begin{aligned}
g_{1}(y)= & c_{1} y^{-m / 2} M\left(\frac{-m}{2 q}-\frac{N_{a}}{2 q^{2}}, 1-\frac{m}{q}, y\right)+c_{2} y^{m / 2} M \\
& \times\left(\frac{m}{2 q}-\frac{N_{a}}{2 q^{2}}, 1+\frac{m}{q}, y\right),
\end{aligned}
$$

where $c_{1}$ and $c_{2}$ are constants and $M$ is the Kummer function [32]. Equation (29) has a regular singularity at $y=0$ and an essential singularity at $y=\infty$. The regular singularity at $y$ $=0$ is removed by the selection $c_{1}=0$ for $m>0$ and $c_{2}=0$ for $m<0$. The outer radial boundary condition for $g_{1}(y)$ at $y=Y$ is determined using $\lim _{Y \rightarrow \infty} l_{i 1}(Y)=0$ together with Eq. (25), giving $\lim _{Y \rightarrow \infty} g_{1}(Y)=0$. Applied to the solution given by Eq. (29) we find [32]

$$
\begin{aligned}
\lim _{Y \rightarrow \infty} g_{1}(Y)= & \frac{\Gamma\left(1+\frac{|m|}{q}\right)}{\Gamma\left(\frac{|m|}{2 q}-\frac{N_{a}}{2 q^{2}}\right)} \lim _{Y \rightarrow \infty}\left(e^{Y} Y^{|m|(q-1) / 2 q-N_{a} / 2 q^{2}-1}\right) \\
& \times\left[1+O\left(|Y|^{-1}\right)\right] \\
= & 0
\end{aligned}
$$

which yields the eigenvalue equation

$$
N_{a}=q(2 q n+|m|),
$$

where $n$ is a positive integer. That is, we select $N_{a}$ to choose the poles of $\Gamma\left(|m| / 2 q-N_{a} / 2 q^{2}\right)$, and so force the coefficient of the limit on the right-hand side to zero. For $q=1$, Eq. (31) reverts to the dispersion relation first derived by Rosenbluth, Krall, and Rostoker [33], which was derived by eliminating the essential singularity in $g_{1}(y)$ at infinity. In the present case, the weaker boundary condition of Rosenbluth, Krall, and Rostoker [33] has the same consequence as the stronger boundary condition used here.

Using [32], solutions to $g_{1}(y)$ for finite $y$ can be expressed as

$$
g_{1}(y) \propto y^{|m| / 2} L_{n}^{(|m|)}(y),
$$

where $L_{n}^{(|m|)}(y)$ are the generalized Laguerre polynomials. The dispersion relation $\varpi=\varpi_{M}(m)$ is given by the solutions of Eq. (31). Including the boundary $\lim _{Y \rightarrow \infty} g_{1}(Y)=0$, the function $g(y)$ given by Eq. (32) has $n+1$ zeros, and so $n$ is a radial eigenmode number.

For these modes, the only experimental parameter required to calculate the normalized growth rate and frequency for different eigenmodes $m$ and $n$ is the normalized plasma rotation frequency $\Omega_{i 0}$. Earlier experiments $[2,3,5,14]$ confirmed that the angular frequency deduced from probe measurements, which is actually the instability frequency $\omega_{i c}$, is comparable to the plasma angular rotation frequency $\Omega_{i 0} \omega_{i c}$. Rather than solving the more complicated inverse problem, we use the measured frequencies.

In the experiments described in Sec. VI with a magnesium plasma, a frequency of $184 \mathrm{krad} \mathrm{s}^{-1}$ was deduced for a magnetic field strength of $B_{z}=0.05 \mathrm{~T}$. Using the value $Z=1.5$ for a magnesium plasma [2] gives $\omega_{i c}=295 \mathrm{krad} \mathrm{s}^{-1}$ and $\Omega_{i 0}=0.59$.

For $q=1$ and $\Omega_{i 0}=0.59$, Figs. 2(a) and 2(b), respectively, plot the normalized growth rate $\varpi^{i}$ and slip $s$, for different azimuthal modes $m$, as a function of the radial eigenmode $n$. The slip is defined as the ratio of the difference between the instability and rotation frequencies with respect to the rotation frequency. That is,

$$
s=\frac{\omega-\Omega_{i 0}}{\Omega_{i 0}} .
$$

As $n$ is integral, only the discrete points in Fig. 2 are solutions of the dispersion relation. As found in earlier treatments [27,33], Fig. 2(a) shows that the $n=0$ solution is stable for $m=1$, and as $m$ increases, the most unstable radial eigen- 

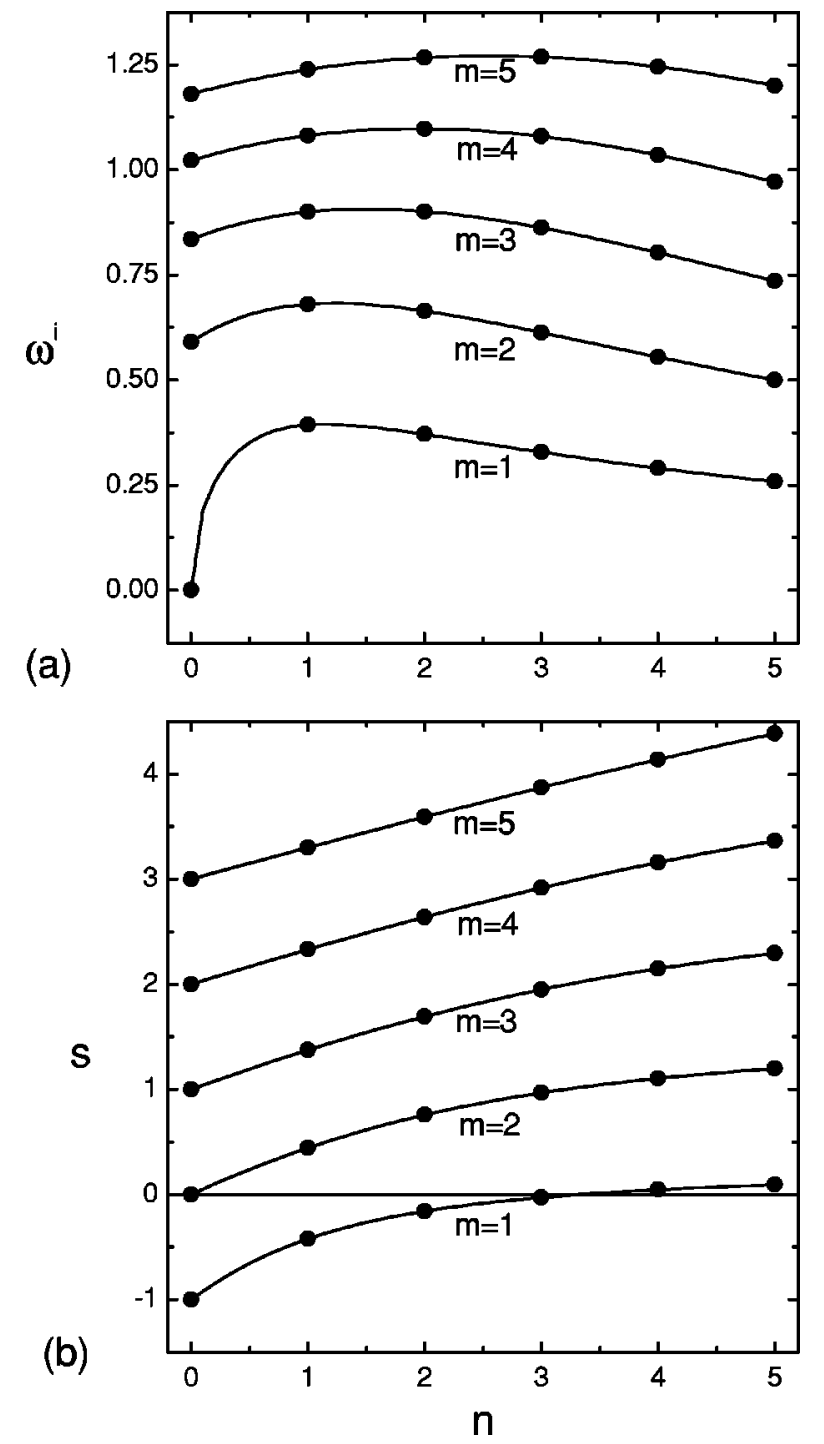

FIG. 2. Flute mode dispersion relation for different $(m, n)$ modes as a function of radial eigenmode number $n$, for normalized plasma rotation frequency $\Omega_{i 0}=0.59$. (a) Plot of the normalized growth rate $\omega^{i}$ and (b) plot of the slip in the laboratory frame $s=\left(\omega-\Omega_{i 0}\right) / \Omega_{i 0}$.

mode increases. It can, furthermore, be shown that providing the normalized rotation frequency $\Omega_{i 0}<0.93$, the $n=1$ radial eigenmode is most unstable [28]. While not included here, higher-order flute eigenmodes are known to be stable in the presence of FLR effects (for $q=1$ ), which limit the set of unstable eigenmodes by limiting the combination $N=2 n$ $+|m|[27,33]$. Figure 2(b) shows that for the $n=1$ eigenmode with $\Omega_{i 0}=0.59$, a slip of $-42 \%$ is predicted, and so the instability is predicted to lag rotation. These modes are the "centrifugal instabilities" [21] and are driven unstable by a difference between the azimuthal drift velocities of the ions and electrons due to a centrifugal force acting on the ions [34].

It will be shown later that an $m=1$ finite axial wavelength mode has a growth rate comparable to the centrifugal instability with $m=1, n=1$. The slip for the finite axial wavelength mode is around $20 \%$, which is much closer to the experimental observations. Furthermore, centrifugal instabilities would have to be present at the anode mesh, which short circuits potential oscillations. Thus, the centrifugal instability is not considered to be a likely explanation for the observed oscillations.

\section{B. Other modes}

For these solutions, $X_{1}(y)=0$ and $l_{i 1}(y)=\chi_{1}(y)$, and the ion density and potential are in phase, which suggests that these waves may be neutrally stable. Eliminating $\varphi_{i 1}(y)$ using the first-row equation of Eq. (23), the second-row equation of Eq. (23) yields the second-order differential equation

$$
\left(\frac{\varpi^{2}-C^{2}}{\varpi}\right) L\left(N_{K}\right)\left[l_{i 1}\right]=0,
$$

where

$$
N_{K}=\frac{\left(\varpi^{2}-k_{z}^{2} \Psi\right)\left(\varpi^{2}-C^{2}\right)}{2 \Psi \varpi^{2}}+\frac{m C q y^{q-1}}{\varpi} .
$$

As with flute modes, the stable zeros of Eq. (34) at $\varpi=$ $\pm C$ are discarded. Equation (34) is solved for $q=1$ only, for which $N_{K}$ reduces to a constant $N_{b}$. As with flute modes, application of the boundary condition $\lim _{Y \rightarrow \infty} l_{i 1}(Y)=0$ yields the eigenvalue equation

$$
N_{b}=2 n+|m|
$$

We have verified numerically that solutions to Eq. (34) are stable for all reasonable VAC plasma conditions.

\section{PERTURBATION SOLUTIONS WITH ELECTRON-ION COLLISIONS}

Amongst other effects, the inclusion of electron-ion collisions is likely to give rise to a universal drift mode [27]. To investigate the excitation of this mode, we investigate small values of $k_{z}$ such that

$$
k_{z}^{2} \lesssim \delta
$$

Introducing $F=k_{z} / \sqrt{\delta}$ and solving for $u_{e z 1}(y)$ from the fifth row of Eq. (20), Eq. (20) can be rewritten as

$$
\left(\begin{array}{c}
\Psi\left[l_{i 1}^{\prime}(y)-X_{1}^{\prime}(y)\right] \\
y \varphi_{i 1}^{\prime}(y) \\
y \varphi_{e 1}^{\prime}(y)-i m \Psi X_{1}^{\prime}(y) \\
\Psi \delta \xi_{\perp} e^{-y^{q}} X_{1}^{\prime}(y)
\end{array}\right)=\widetilde{\mathbf{A}}_{1}\left(\begin{array}{c}
l_{i 1}(y) \\
X_{1}(y) \\
\varphi_{i 1}(y) \\
\varphi_{e 1}(y)
\end{array}\right)
$$




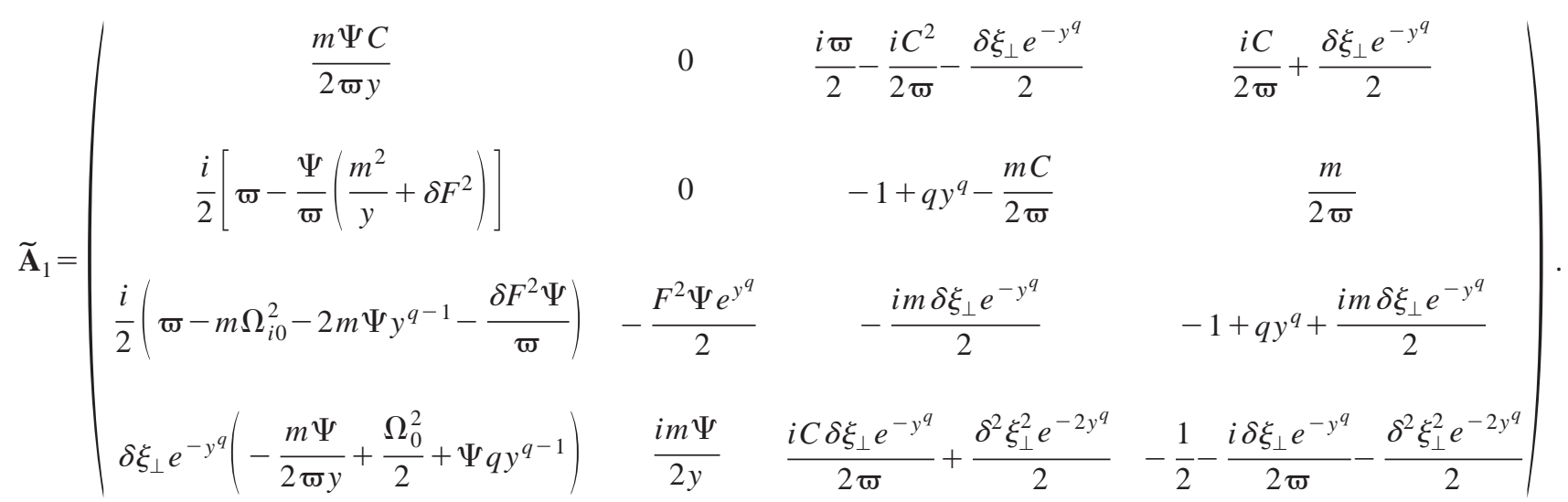

Applying the inequality of Eq. (36) implies $F^{2} \leq 1$. For typical VAC conditions $\delta \ll 1$ and so we ignore all terms of $O(\delta)$ in Eq. (37). When this assumption is made, $F^{2}$ vanishes from all but the $X_{1}(y)$ coefficient in the third-row equation in Eq. (37). The restriction $F^{2} \leqslant 1$ together with the neglect of $O(\delta)$ terms in Eq. (37) can be shown [28] to be equivalent to two alternative assumptions: (i) neglect of the effects of perpendicular collisions and (ii) neglect of the perturbation in the ion axial velocity, which were made by Chen [27]. Both assumptions (i) and (ii) are used in the theory of drift waves in the absence of classical diffusion [26].

When $\Psi=0, X_{1}(y)$ and $X_{1}^{\prime}(y)$ vanish in Eq. (37), and solutions for $l_{i 1}(y)$ are neutrally stable. We, therefore, restrict attention to a warm plasma, and follow the same approach as Sec. IV. Expressions for $\varphi_{i 1}(y)$ and $\varphi_{e 1}(y)$ are found from the first and fourth rows of Eq. (37), respectively. Next, $X_{1}(y)$ is replaced with the dependent variable $g_{1}(y)$ $=X_{1}(y)-l_{i 1}(y)$, and the third row of Eq. (37) is solved for $l_{i 1}(y)$, giving

$$
l_{i 1}(y)=\frac{-g_{1}(y)}{1+\frac{i}{\Psi}\left(\frac{m \Omega_{i 0}^{2}+2 m \Psi q y^{q-1}-\varpi}{f(y)-2 i m q y^{q-1}}\right)} .
$$

Finally, expressions for $\varphi_{e 1}(y), \varphi_{i 1}(y), X_{1}(y)$, and $l_{i 1}(y)$ are substituted into the second row of Eq. (37), giving the second-order differential equation

$$
\left(\frac{\varpi^{2}-C^{2}}{\varpi \Psi}\right) L\left(N_{c}\right)\left[g_{1}(y)\right]=0
$$

where

$$
N_{c}=\frac{\left(\varpi^{2}-C^{2}\right)\left[m q y^{q-1}+\frac{i}{2} f(y)\right]}{\left[\varpi-m \Omega_{i 0}^{2}+i \Psi f(y)\right]}+\frac{m q C}{\varpi} y^{q-1}
$$

and $f(y)=k_{z}^{2} e^{y^{q}} / \delta=F^{2} e^{y^{q}}$. As for the cases without collisions, the stable zeros of Eq. (34) at $\varpi= \pm C$ are discarded. When $k_{z}=0$, the expression for $N_{c}$ [Eq. (41)] reduces to the expression for flute modes $N_{a}$ [Eq. (28)], and the expression for $l_{i 1}(y)$ [Eq. (39)] reduces to that for flute modes [Eq. (25)]. That is, with the assumptions made here, collisions do not affect the flute modes.

Equation (40) can be solved analytically when $N_{c}$ is a constant. This occurs when $q=1$ and either $\varpi= \pm C$ (already discarded), $f(y)=0$, or $f(y) \rightarrow \infty$. The case $f(y)=0$ requires $k_{z}=F=0$ (flute modes). Alternatively, the case $f(y) \rightarrow \infty$ requires $F \rightarrow \infty$, and so $k_{z} \rightarrow \infty$ for which the inequality in Eq. (36) fails, or $\delta \rightarrow 0$, solutions of which were investigated in Sec. IV.

When $k_{z} \neq 0$, Eq. (40) was solved numerically for $q=1$. For odd $m$, the solution for $l_{i 1}$ is an odd function of the radius and thus $l_{i 1}(y)$ and $g_{1}(y)$ are both zero on-axis. For even $m$, the solution for $l_{i 1}$ is an even function of radius and thus $l_{i 1}^{\prime}(x=0)=0$. As with odd $m$ values, the on-axis boundary condition for even $m$ values in $g_{1}(y)$ is found to be homogeneous.

In the numerical calculation, the boundary condition at infinite radial distance was replaced with $g_{1}(y)=0$ at $y$ $=Y$. The initial slope at $y=Y, g_{1}^{\prime}(Y)$, is arbitrary since the differential equation, Eq. (40), and the homogeneous boundary conditions only determine $g_{1}(y)$ to within a complex multiplicative constant. For a given value of $F$, Eq. (40) was then integrated inwards to $y=0$. A search algorithm [28] was used to find values of $\varpi^{r}$ and $\varpi^{i}$ to match the homogeneous on-axis boundary condition. Of these solutions, modes with maximum growth (maximum $\varpi^{i}$ ) could be found by using a suitable search method [28].

Use was made of the analytical solutions at $F=0$ or $F$ $\rightarrow \infty$ to provide a numerical check on the accuracy of the simulations. For finite $Y$ and $q=1$ the exact solution can be generated by solving $g_{1}(Y)=0$ for $N$ [35], where

$$
g_{1}(Y) \propto Y^{|m| / 2} M\left(\frac{|m|}{2}-\frac{N}{2}, 1+|m|, Y\right),
$$

and then using Eq. (41) with $F=0$ or $F \rightarrow \infty$ to solve for $\varpi^{r}$ and $\varpi^{i}$. These checks were used to confirm the validity of the numerical solution, which was found accurate to within three significant figures at $F=0$ and $F=10$. 
To be certain that the dispersion solution from both codes represented the behavior of a free plasma column, the outer radial boundary was moved out until numerical convergence of the dispersion relation could be established. The values presented in this work are calculated with $Y=20$. No change in the dispersion relation could be observed when $Y$ was decreased to 16 . Finally, the exact solution at $k_{z}=0$ with the boundary condition at infinity provides a check of convergence of the numerical solution.

To completely specify the solution, the normalized plasma rotation frequency $\Omega_{i 0}$ and normalized thermal velocity $\Psi$ are required [see Eqs. (40) and (41)]. As for Sec. IV A $\Omega_{i 0}$ $=0.59$. In the experiments described in Sec. VI radial profiles of the electron temperature and ion density profile are reported for a magnesium plasma. An estimate of the electron temperature $T_{e}=2.9 \mathrm{eV}$ comes from an average of the profile across the bulk of the plasma column, whilst the characteristic radius $R=14.3 \mathrm{~mm}$ is inferred from a Gaussian fit to the ion density profile. Assuming equal ion and electron temperatures, a value of $\Psi=1.6$ is estimated for a magnesium plasma.

For $m=0$ and $F \leqslant 1$ no unstable solutions were found with $\left|\varpi^{r}\right| \leqslant 1$. Figure 3 is a dispersion curve of $\varpi^{r}$ and $\varpi^{i}$ for the most unstable $m=1$ modes as a function of $F$. Because $\varpi$ is an even function of $F$, curves are restricted to $F$ $\geqslant 0$. Figure 3 (a) shows the instability growth rate $\varpi^{i}$ and Fig. 3(b) shows the frequency in the plasma frame $\varpi^{r}$. In Fig. 3(b), the regions $m=+1$ and $m=-1$ correspond to wave propagation in the $+\boldsymbol{\theta}$ and $-\boldsymbol{\theta}$ directions, respectively. The heavy curve in both figures is the most unstable solution. To show mode features, modes have been tracked beyond the intervals in $F$ over which they are most unstable. At $F$ $=0.13$, a change in the most unstable mode occurs. At $F$ $=0.55$ a mode crossover occurs, where the frequency and growth rate of two modes cross. The crosses at $F=0$ mark the standing wave flute mode solutions for $n=0$ and $n=1$ in the limit $Y \rightarrow \infty$.

The growth rates near $F=0$ and $F=0.3$ are comparable. Properties of the $k_{z}=F=0$ centrifugal instability were discussed in Sec. IV. The solution for $m=1$ at $F=0.3$ is a density-gradient driven drift wave with the following features.

(a) The value of $F \simeq 0.3$ for peak growth is reasonably insensitive to the variation of $C$ and $\Psi$ over the range 1 $<C<3$ and $0.01<\Psi<2$. Using $F=0.3$ and $\delta=0.03$ (for the magnesium plasma treated here) an axial wavelength of $\lambda_{z}=1.7 \mathrm{~m}$ is calculated.

(b) The plasma frame frequency $\varpi^{r} \simeq 0$ and so the wave is stationary in the plasma frame.

(c) Perturbations in the ion density and ion radial velocity are in phase to within $20^{\circ}$ across the bulk of the plasma. In regions of increased perturbed density $n_{i 1}$, radial outflow of the plasma occurs, bringing in plasma from a region of larger steady-state density $n_{i 0}$ to increase $n_{i}$ further. In regions of decreased $n_{i 1}$, radial inflow of the plasma occurs, bringing in plasma from a region of lower $n_{i 0}$ to decrease $n_{i}$ further. The oscillations are driven by the density gradient.
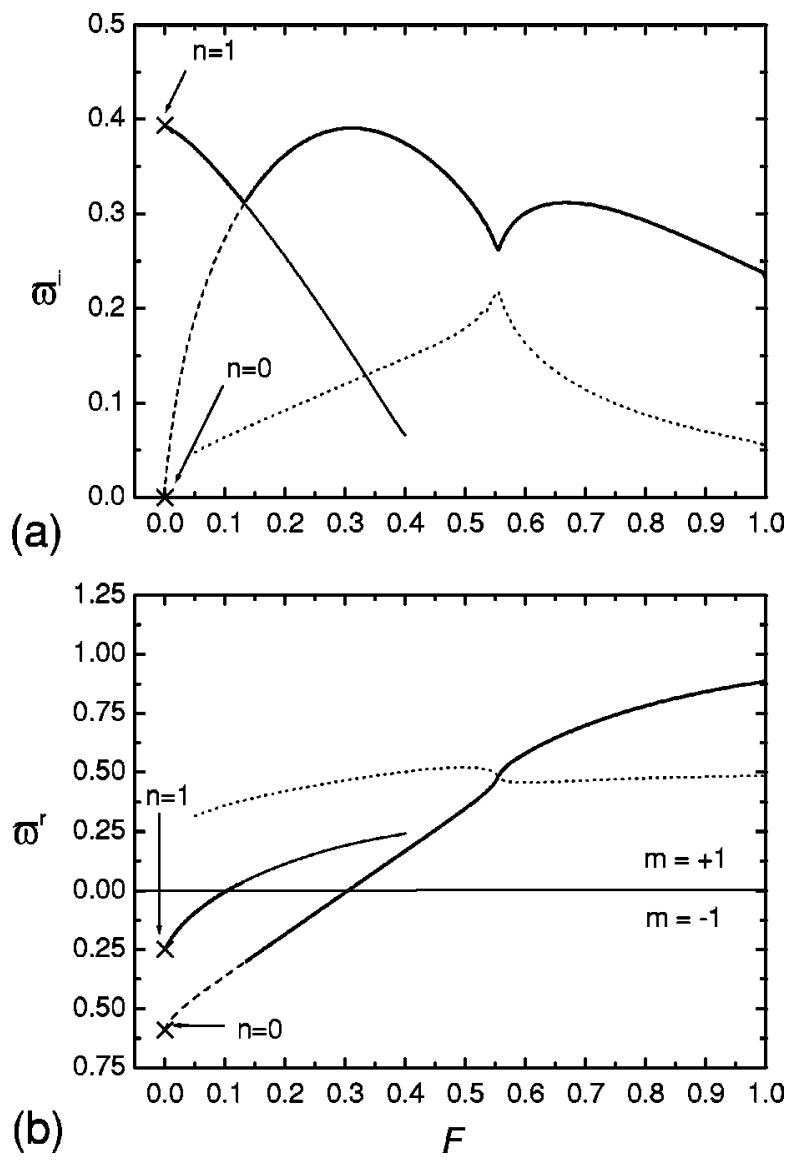

FIG. 3. Dispersion curves of (a) the normalized growth rate $\varpi^{i}$ and (b) the normalized frequency in the frame of the ion fluid $\boldsymbol{\varpi}^{r}$, for $m=1$ plotted against normalized axial wave number $F$. Plasma conditions are taken from Table I. The solid line is the most unstable mode envelope, and the crosses represent the eigenmodes of the centrifugal instability of an unbounded plasma column $(Y \rightarrow \infty)$.

(d) The phase difference between the ion density and potential is close to $180^{\circ}$ across the bulk of the plasma column. The presence of a phase difference between the ion density and potential is a necessary condition for the drift wave to be unstable $[26,36]$.

(e) The phase of the ion density is constant across the bulk of the plasma, meaning that within this region the wave is standing in the radial direction.

The instability at $F=0.3$ is the "universal" instability of a plasma with the finite conductivity of Chen [26,37], driven by the density gradient, but here modified by rotation $[27,36]$ and with laboratory frame oscillation frequency $\omega$, Doppler shifted due to the ion axial streaming velocity.

For finite axial wavelength, calculation of the slip requires an estimate of the ion axial velocity. In this work the ion axial velocity $v_{z 0}$ was not measured, and so a value of $10^{4} \mathrm{~m} \mathrm{~s}^{-1}$ has been taken for the magnesium plasma, based on measurements by Dallaqua et al. [2]. Using $\omega_{i c}$ $=295 \mathrm{krad} \mathrm{s}^{-1}$ and $R=14.3 \mathrm{~mm}$ an estimate for the normalized ion axial velocity $u_{* 0}=v_{* 0} / \omega_{i c} R=2.37$ is inferred. Figure 4 shows the calculated slip of the most unstable solution for $m=1$ as a function of $F$. The slip is not an even function 


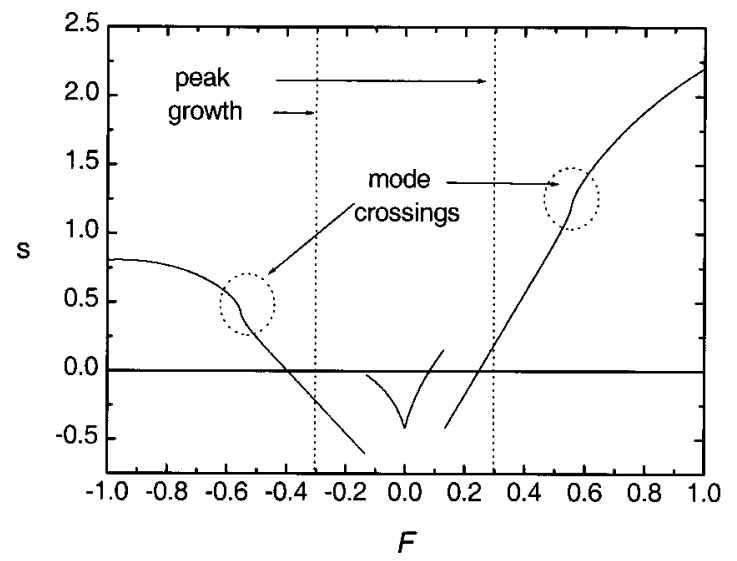

FIG. 4. Laboratory frame slip $s=\left(\omega-\Omega_{i 0}\right) / \Omega_{i 0}$ for the most unstable $m=1$ mode versus normalized axial wave number $F$.

of $F$ and so the curves have been extended to include negative $F$ values. The effects of the mode change at $F=0.13$ cause the discontinuity in the slip curve, whilst the mode crossing at $F=0.55$ is responsible for the kink. Over the range $|F|<1, s>-1$ indicating that the azimuthal component of wave propagation is always in the direction of rotation. The dotted lines correspond to peak growth at $|F|$ $=0.3$. The wave is close to stationary in the frame of the ion fluid. Thus, in the laboratory frame, the wave fronts propagate downstream (i.e., $k_{z}>0$ ), and so $F=k_{z} / \sqrt{\delta}>0$. Finally, referring to Fig. 4 , a slip of $s \simeq 0.21$ is, therefore, predicted for these modes.

Figure 5 is a dispersion curve of $\varpi^{r}$ and $\varpi^{i}$ for the most unstable $m=2$ modes as a function of $F$. As with $m=1$, the heavy solid line is the most unstable solution, and the crosses mark the $n=0$ and $n=1$ flute modes in the limit $Y \rightarrow \infty$. In general, the features of the $m=1$ and $m=2$ dispersion curves are similar. For $m=2$, however, all modes are more unstable and the flute mode has the largest growth rate.

As noted earlier, for $q=1$, FLR effects are known to stabilize higher-order flute modes by limiting the combination $N=2 n+|m|$. For a $Q$ machine plasma, stability of the density-gradient driven drift wave has been investigated by Chu et al. [36] in a fluid treatment, which includes both ion collisional viscosity and FLR effects. Their findings suggest that as $m$ increases, the $k_{z}$ interval over which the drift wave is unstable decreases, and that this effect is due to ion collisional viscosity. For the VAC plasma, a detailed stability analysis, which includes both FLR effects and ion collisional viscosity, is necessary to properly describe stabilization. However evidence for a similar type of plasma suggests stabilization of higher-order density-gradient driven drift modes [36].

Finally, a simple estimate of the growth of the wave can be made over the time taken for the wave front to propagate from the anode mesh to the end plate, a distance of about 1 $\mathrm{m}$ in the PCEN device. Using an initial growth rate of $\varpi^{i}$ $\approx 0.4$ for the $m=1$ density-gradient driven drift wave at $F$ $=0.3$, it can be shown [28] that from anode mesh to end plate the wave amplitude increases by the large factor $e^{12}$; and thus will most likely be in a saturated state at the end
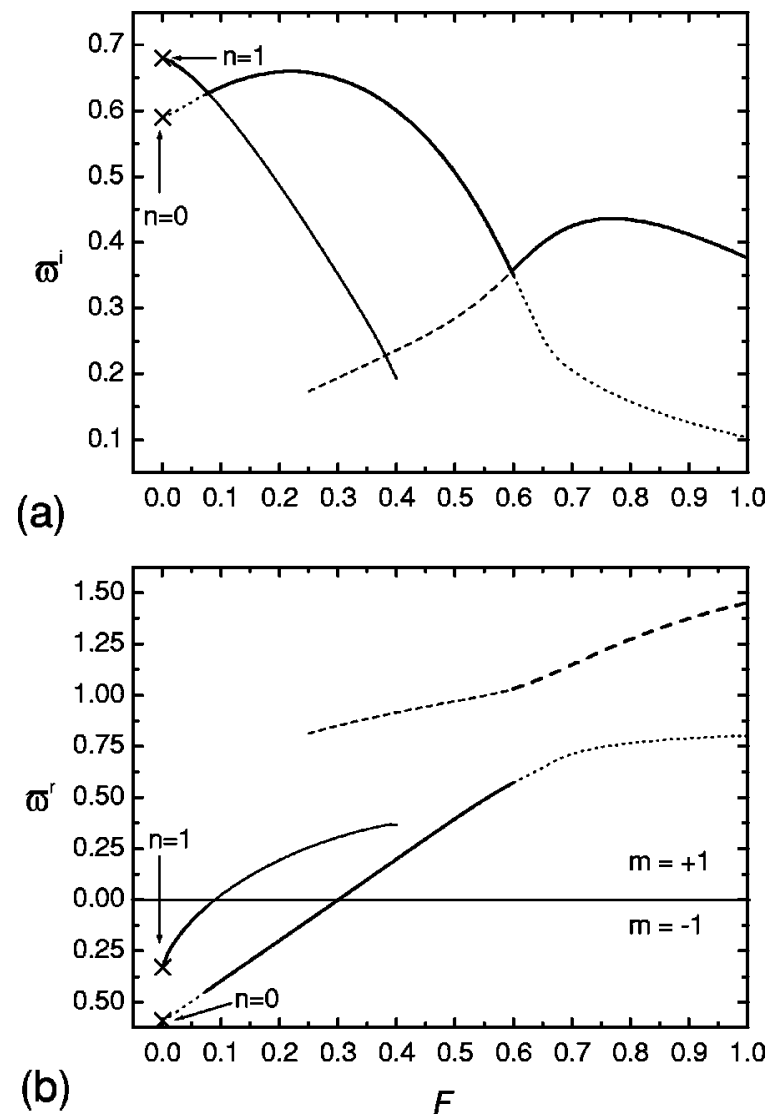

FIG. 5. Dispersion curves of (a) the normalized growth rate $\varpi^{i}$ and (b) the normalized frequency in the frame of the ion fluid $\varpi^{r}$, for $m=2$ plotted against $F$. Plasma conditions are taken from Table I. The solid line is the most unstable mode envelope and the crosses represent the eigenmodes of the centrifugal instability of an unbounded plasma column $(Y \rightarrow \infty)$.

plate. A more complete analysis of the temporal evolution of the wave amplitude requires a more sophisticated plasma model, which takes into account the dynamics of saturation.

\section{THE EXPERIMENT}

Experiments were conducted on the PCEN [2] device at the Brazilian National Space Research Institute, which is a typical VAC. The PCEN device is operated with a $1 \mathrm{kA}$ discharge current lasting for $10-15 \mathrm{~ms}$ and $B_{z}<0.2 \mathrm{~T}$, which is constant in the rotation region to within 5\%. Figure 1 shows schematics of (a) the PCEN device and (b) the magnesium cathode used for most measurements. To avoid preferential emission from the edges of the cathode, a highvoltage trigger electrode was placed on-axis by boring a hole through the cathode center. In all experiments the anode was a tungsten grid with about $60 \%$ transparency, placed $15 \mathrm{~mm}$ from the cathode surface. Preliminary mylar film plasma deposition experiments [28] showed that over the range 0 $<z<0.45 \mathrm{~m}$, the plasma column center lay within $1.6 \mathrm{~mm}$ of the vessel axis [28].

In the experiments reported here, extensive measurements were made with Langmuir probes in the (5-12)-ms interval following discharge. These measurements were used to de- 

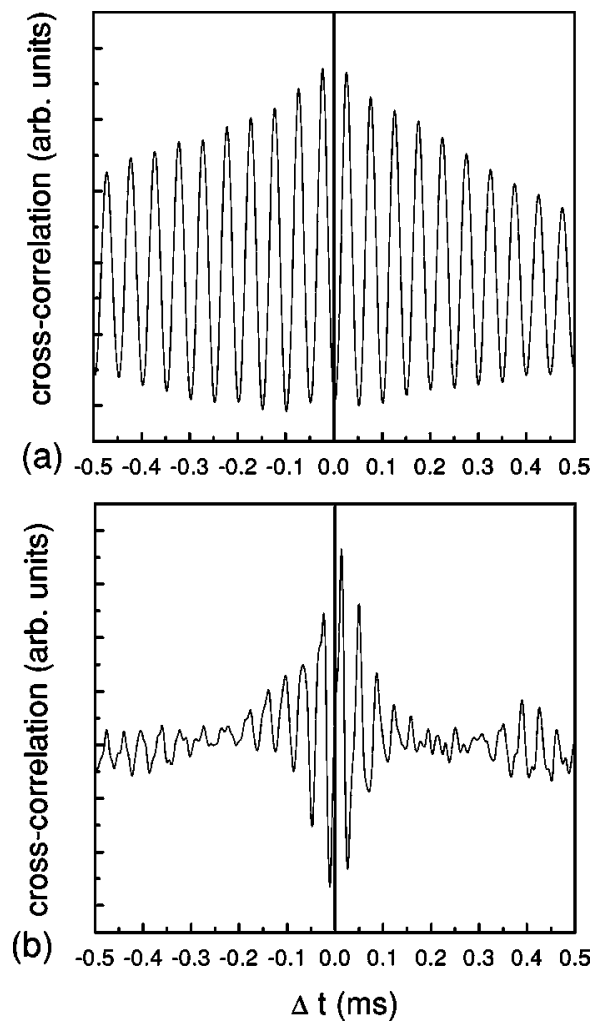

FIG. 6. Plots of the cross correlation of the floating potential measured from two probes located at the same axial position (150 $\mathrm{mm}$ from the anode mesh) and separated by $180^{\circ}$ in azimuth. In (a) the probes were located at a radial distance of $r=22 \mathrm{~mm}$ from the geometric center. In (b) the probes were located at radial distances of $r=5$ and $35 \mathrm{~mm}$

duce ion densities, electron temperatures, and also to investigate the phase relationship between instability oscillations observed at different locations.

The ion density was estimated from the ion saturation current with a bias voltage in the range -50 to $-70 \mathrm{~V}$, following the technique described in Ref. [2]. The cylindrical probe collection area was $2 \mathrm{~mm}$ long with diameter $0.2 \mathrm{~mm}$.

The electron temperature was deduced in the usual way by sweeping the probe bias voltage $[2,12,14,28]$. Previous measurements $[2,12]$ have shown that there is little variation in electron temperature throughout the discharge.

Following earlier work $[6,12,14]$, cross correlation of probe signals was used to deduce phase relationships for both the floating potential signals and the ion saturation current. The data window was of $4.5 \mathrm{~ms}$ and eight runs were averaged over. As for previous work [12] it was found that there was little variation in phase relationships through the course of the discharge.

Figure 6(a) shows a typical cross correlation of floating potential signals. The probes were positioned at the same axial position ( $150 \mathrm{~mm}$ from the anode grid) and radius $(22$ $\mathrm{mm}$ ), but separated by $180^{\circ}$ in azimuth. It can be seen that the peaks in the correlation are well defined, with the offset of the closest peak from zero giving the phase difference and the peak spacing giving the angular frequency $[6,12]$.

Figure $6(\mathrm{~b})$ shows a correlation of signals also at the same axial position and separated in azimuth by $180^{\circ}$, but with one probe at $r=5 \mathrm{~mm}$ and the other at $r=35 \mathrm{~mm}$. It can be seen that the maxima near zero time are well defined, however there is an overall low-frequency modulation of the maxima likely to be associated with a small change in angular frequency of the instability between the two radial positions. This deviation from rigid body rotation is not considered in this work.

For the measurements at a given radial location described above, the probes, which have a 1.6-mm-diameter ceramic sleeve, were inserted radially into the plasma column. For comparisons of different axial locations, probes were inserted axially using guides outside the plasma column (see Fig. 1). These probes were bent at right angles $85 \mathrm{~mm}$ from the tip to enter the plasma column almost radially.

For the experimental radial phase profiles presented in the following section, a fixed probe measuring the floating potential provided a phase reference. The reference probe, located at $z=0.2 \mathrm{~m}$, was displaced $50 \mathrm{~mm}$ downstream from the measurement plane at $z=150 \mathrm{~mm}$. The radial position of the reference probe was $20 \mathrm{~mm}$, and the probe was located on the opposite side of the column at an azimuthal position $180^{\circ}$ from the measurement probe. This arrangement was chosen to minimize plasma perturbation and any possible interaction between the probes.

For the experimental axial phase profile presented in the following section a slightly modified cathode was used, with the high-voltage trigger positioned on the ceramic isolator. For the axial phase profiles, one probe was fixed at an axial position of $z=60 \mathrm{~mm}$, and the position of the second probe varied between $z=0.06 \mathrm{~m}$ and $z=0.31 \mathrm{~m}$. The probes were positioned at the same radius ( $r=22 \mathrm{~mm}$ ), but separated by $180^{\circ}$ in azimuth.

\section{RESULTS AND DISCUSSION}

Figure 7 shows measured radial profiles of (a) the steadystate ion density, (b) electron temperature and total pressure, and (c) instability frequency for the plasma with $B_{z}$ $=0.05 \mathrm{~T}$ at $z=150 \mathrm{~mm}$. A Gaussian profile has been fitted to the ion density curve, and it well fits the data. From Fig. 7(a) it can be seen that the assumption that the electron temperature is uniform is poor outside the bulk of the plasma column. The pressure profile [Fig. 7(b)], calculated by $p$ $=n_{i} k_{B}\left(T_{i}+Z T_{e}\right)$ with $T_{i}=T_{e}$, is not Gaussian owing to the nonuniformity of $T_{e}$. Finally the rotation frequency [Fig. 7 (c)] is uniform with the radius, which is consistent with the VAC plasma model. From the profiles in Fig. 7, the operating conditions shown in Table I were deduced.

Figures 8 and 9 show the radial phase variation of the ion density and floating potential for the experimental data and theory. The theory curves in Figs. 8 and 9 are for the $n=1$ centrifugal instability and the density-gradient driven drift wave, respectively. Figure 10 shows the measured and predicted phase difference between the ion density and potential oscillations. It should be mentioned that, in Figs. 8 and 9, phase angles have not been restricted to a $360^{\circ}$ range. Leaving the plots to continuously cover a larger range allows trends in the experimental data and theory to be clearly iden- 


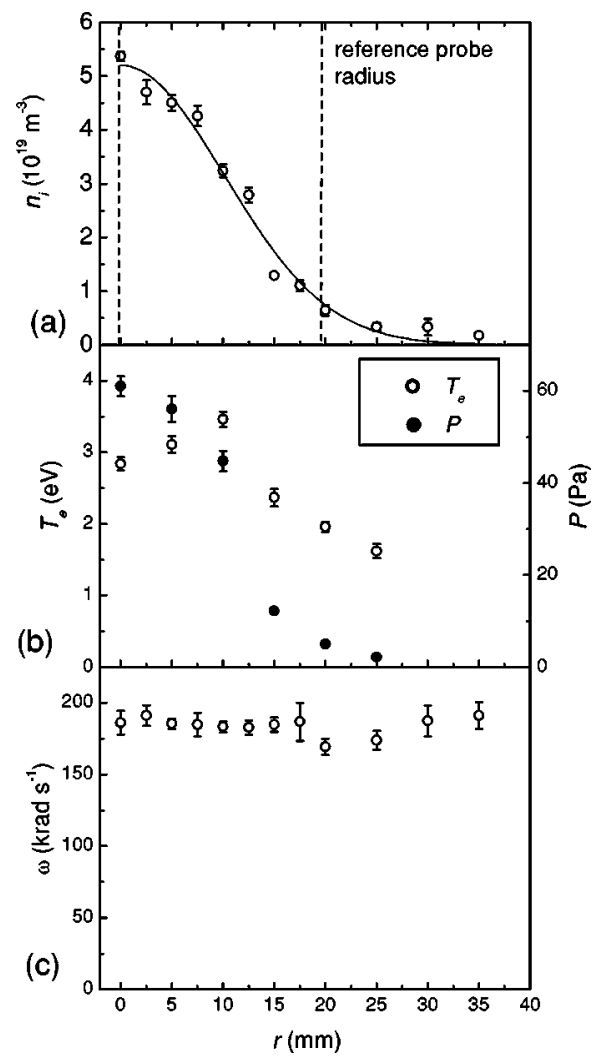

FIG. 7. Radial profiles of (a) ion density $n_{i}$, (b) electron temperature $T_{e}$ (left axis) and total pressure $P$ (right axis), and (c) instability frequency $\omega$ with $B_{z}=0.05 \mathrm{~T}$.

tified. In Figs. 8 and 9, the predicted phase of the floating potential as $r \rightarrow 0$ has been arbitrarily taken as reference. There is an arbitrary phase difference between theory and experiment, which has been eliminated by adjusting the

TABLE I. Plasma conditions for a magnesium plasma.

\begin{tabular}{ll}
\hline \hline Parameter & Value \\
\hline$B_{z 0}$ & $0.05 \mathrm{~T}$ \\
$\omega_{0}$ & $184 \mathrm{krad} \mathrm{s}^{-1}$ \\
$n_{i 0}$ & $5.2 \times 10^{19} \mathrm{~m}^{-2}$ \\
$R$ & $14.3 \mathrm{~mm}$ \\
$T_{e}$ & $2.9 \mathrm{eV}$ \\
$v_{z 0}{ }^{\mathrm{a}}$ & $10 \mathrm{~km} \mathrm{~s}^{-1}$ \\
$Z^{\mathrm{a}}$ & 1.5 \\
$\omega_{i}=\frac{B_{z} e Z}{M}$ & $295 \mathrm{krad} \mathrm{s}^{-1}$ \\
$\Omega_{0}=\frac{\omega_{0}}{\omega_{i}}$ & 0.59 \\
$\psi=\frac{k_{B} T_{e}}{M \omega_{i c}^{2} R^{2}}$ & 0.64 \\
$\delta=\frac{e Z n_{i 0}}{B_{z}} \frac{\eta_{L}}{\gamma_{E}}$ & 0.03 \\
\hline
\end{tabular}

${ }^{\mathrm{a}}$ Taken from [2].

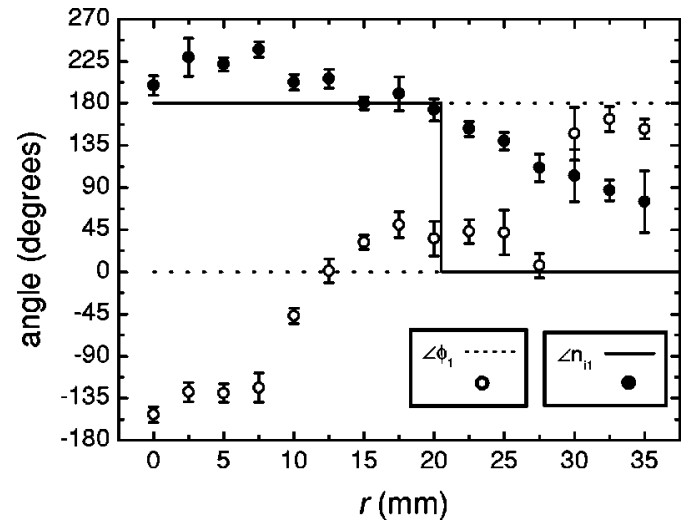

FIG. 8. Plot of the radial phase profile of the floating potential and ion density, fitted such that $\angle n_{i}=180^{\circ}$ at $r=15 \mathrm{~mm}$. The lines are the predictions for the centrifugal instability $\left(k_{z}=0\right)$ with $m$ $=1, n=1$.

phase of the measured ion density and potential oscillations, so that in both Figs. 8 and 9 the phase of the ion density is matched to the predicted phase for the centrifugal and density-gradient driven drift waves at $r=15 \mathrm{~mm}$, respectively. Figure 10 plots differences in phase, and there is no arbitrariness in the phase.

It can be seen in both Figs. 8 and 10 that the phase profile for the $n=1$ centrifugal instability does not agree with experimental data. As mentioned in Sec. IV A, the slip is also rather large at about $-42 \%$ for this mode.

Turning to the density-gradient driven drift wave, agreement is reasonable for both the absolute phase profiles (Fig. 9) and the phase difference between potential and saturation current (Fig. 10) for radii greater than about $10 \mathrm{~mm}$ and less than $25 \mathrm{~mm}$. As mentioned in Sec. V, the predicted slip is about $21 \%$ for this wave.

The predicted and measured phase profiles of the ion saturation current are in reasonable agreement (Fig. 9) for $r$ $<25 \mathrm{~mm}$, with the phase close to constant across the whole column. For $r>25 \mathrm{~mm}$, however, where the ion density has fallen to below $5 \%$ of its on-axis value, the phase of the ion density exhibits poor agreement with predictions of theory.

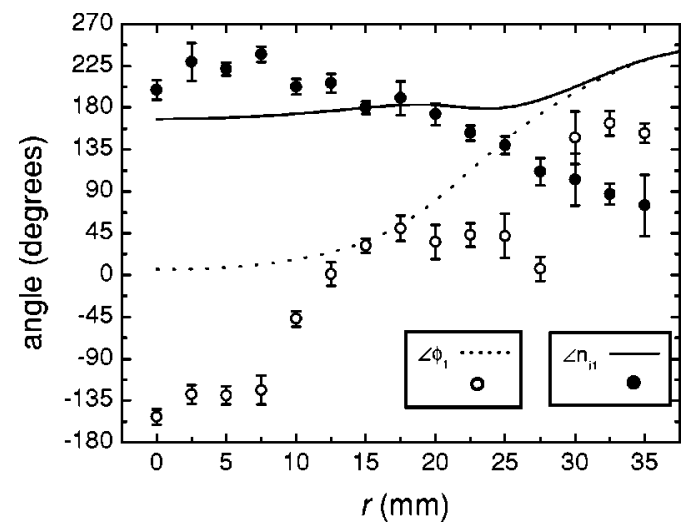

FIG. 9. Plot of the radial phase profile of the floating potential ion density, fitted such that $\angle n_{i}=180^{\circ}$ at $r=15 \mathrm{~mm}$. The lines are the predictions for the density-gradient driven drift mode $\left(k_{z}\right.$ $=3.7 \mathrm{rad} \mathrm{m}^{-1}$ ) with $m=1$. 


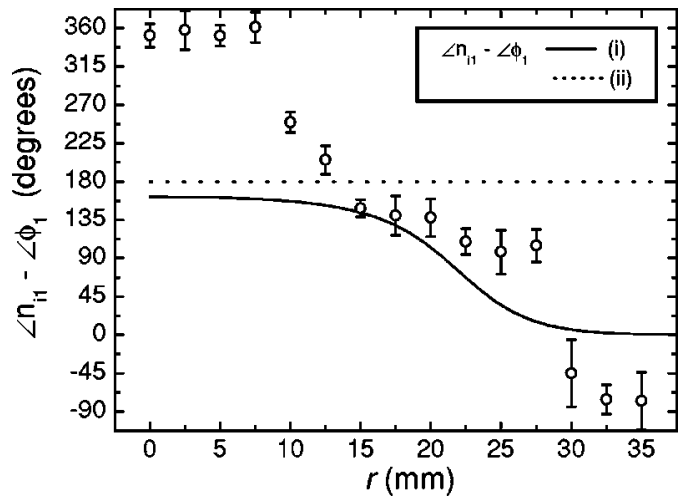

FIG. 10. Radial phase difference between the ion density and floating potential. The lines are the predictions of theory; case (i) is the density-gradient driven drift mode and case (ii) is for the $n$ $=1$ centrifugal instability.

This may relate to probe perturbation effects, or an inaccurate theoretical description of this low density plasma. The phase profile of the floating potential (Fig. 9) and the phase difference (Fig. 10) also exhibit some discrepancy with theory for radii greater than $25 \mathrm{~mm}$.

For radii less than $10 \mathrm{~mm}$, there is a more significant discrepancy between theory and experiment in the profile of the floating potential (Fig. 9) and in the phase difference (Fig. 10). In particular, the measured phase difference between ion density and potential approaches zero $\left(360^{\circ}\right.$ in Fig. 10 ), which is unexpected for a density-gradient driven drift wave $[26,36]$. Possible causes of this discrepancy are an incomplete theoretical description of the plasma, or perturbation of the plasma, when the probe is inserted close to the axis.

Figures 11 and 12 show the radial profile of the measured and predicted amplitudes of the oscillation in both the potential and ion density. The measured amplitudes of the floating potential and ion density oscillations have been normalized to their peak values, respectively. Except the predicted amplitude of the oscillation of the floating potential for the centrifugal mode (Fig. 11), which has been normalized to its maximum value in the range $0<r<20 \mathrm{~mm}$ (i.e., before the node), the predicted amplitude profiles have also been normalized to their peak values, respectively.

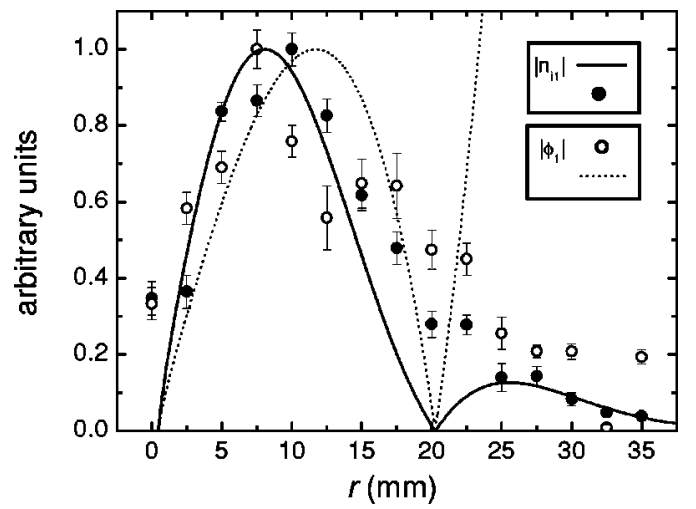

FIG. 11. Radial profiles of $\left|\phi_{1}\right|$ and $\left|n_{i 1}\right|$ with $B_{z}=0.05 \mathrm{~T}$. The lines are the predictions for the $n=1$ centrifugal instability.

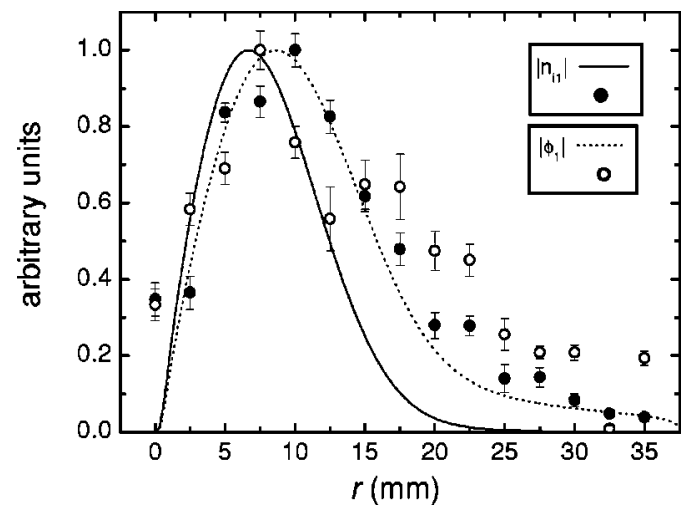

FIG. 12. Radial profiles of $\left|\phi_{1}\right|$ and $\left|n_{i 1}\right|$ with $B_{z}=0.05 \mathrm{~T}$. The lines are the predictions for the density-gradient driven instability.

It can be seen that for the $n=1$ centrifugal instability (Fig. 11), the measured amplitudes agree reasonably with the predicted profiles out to the characteristic radius. However, the $n=1$ eigenmode has a node at $r=20 \mathrm{~mm}$. In contrast, the measured amplitude of the oscillations is nonzero at this location. Beyond the node at $r=20 \mathrm{~mm}$, the predicted amplitude profile of the floating potential oscillation increases significantly. In contrast, the measured amplitude profile decreases with increasing radius.

Turning to the density-gradient driven drift wave (Fig. 12), the observed maxima are close to the predicted radial locations, however the experimental peaks are broader than predictions. This discrepancy may be explained by the fact that the perturbation treatment is first order while the experimental data represents a nonlinear saturated state of the instability.

The instability frequency was measured and was found to be independent of axial position. Figure 13(b) shows the axial profile of the phase difference between floating potential signals $\Delta \Phi$, inferred from the cross correlation. The phase plot shows a $\Delta \Phi \approx 30^{\circ}$ increase across the $0.25 \mathrm{~m}$ length. A line of best fit gives $\Delta \Phi / \Delta z=110 \pm 10 \mathrm{deg} \mathrm{m}^{-1}$, giving $k_{z}=1.9 \pm 0.2 \mathrm{rad} \mathrm{m}^{-1}$. As the gradient is positive, the wave propagates downstream. This compares reasonably to

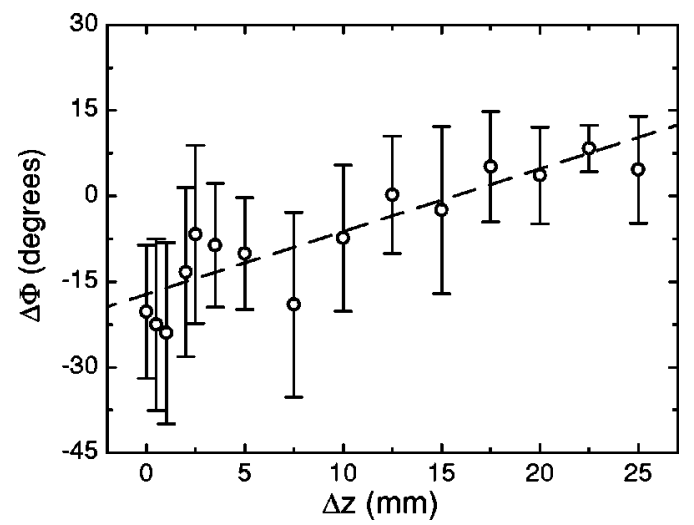

FIG. 13. Phase difference between the floating potential of probes separated by $\Delta z$ and positioned $180^{\circ}$ apart in azimuth. A constant phase of $180^{\circ}$ has been subtracted from the phase measurements. 
the value of $k_{z}=3.7 \mathrm{rad} \mathrm{m}^{-1}$ inferred from theory for the density-gradient driven drift wave.

\section{CONCLUSIONS}

In this work the standard VAC plasma model was linearized with a wave perturbation, solutions found, and dispersion curves generated for a range of conditions. Two unstable modes were found for the VAC plasma: the centrifugal instability $[33,34]$, and the density-gradient driven drift wave $[26,27]$.

Detailed Langmuir probe measurements were made of a magnesium plasma in the PCEN device, a typical VAC. These measurements were used to deduce ion densities, electron temperatures, and also to investigate the phase relationship between instability oscillations observed at different locations, which were then compared to the predictions of theory.

The most likely candidate instability is the densitygradient driven drift wave of a plasma with finite electrical conductivity. The density-gradient driven drift wave with $m$ $=1$ has a predicted slip of $21 \%$. This is to be compared to a slip of $17 \%$ estimated from plasma deposition measurements in a similar magnesium plasma $[2,14]$. The axial wave number of the density-gradient driven drift wave is predicted to be $k_{z}=3.7 \mathrm{rad} \mathrm{m}^{-1}$, which is comparable to the measured wave number of $k_{z}=1.9 \pm 0.2 \mathrm{rad} \mathrm{m}^{-1}$. The predicted radial phase profile of the ion density compares well to the measured profile, and agreement between the predicted and mea- sured phase profile of the floating potential is reasonable for $r>10 \mathrm{~mm}$. For $r<10 \mathrm{~mm}$, however, there is a significant discrepancy in the profile of the floating potential between the predictions of the density-gradient driven drift wave and experiment. It is conjectured that causes of the discrepancy are either an incomplete theoretical description of the plasma or perturbation of the plasma when the probe is located at small radii. Two areas where the theoretical description could be lacking are in the neglect of nonlinear terms and in the assumption of electron temperature invariance across the plasma and during the discharge. Finally, the maxima in the instability amplitude profiles of the floating potential and ion density are close to the predicted radial location.

In conclusion, the theoretical calculations and experimental data reported here indicate that the instability observed in vacuum arc centrifuges since 1980 is a density-gradient driven drift mode (belonging to the class of universal instabilities) in a plasma with finite electrical conductivity.

\section{ACKNOWLEDGMENTS}

The authors gratefully acknowledge the support of the Department of Education, Training and Youth Affairs, the School of Electrical and Information Engineering, and the University of Sydney for the provision of Grants-in-aid that assisted in travel to Brazil, which enabled one of us (M.J.H.) to work on this project. The authors also gratefully acknowledge the financial support of the Research Foundation of the State of São Paulo (FAPESP) towards the PCEN experiment.
[1] M. Krishnan, M. Geva, and J. L. Hirshfield, Phys. Rev. Lett. 46, 36 (1981).

[2] R. S. Dallaqua, E. Del Bosco, R. P. Da Silva, and S. W. Simpson, IEEE Trans. Plasma Sci. 26, 1044 (1998).

[3] M. Geva, M. Krishnan, and J. L. Hirshfield, J. Appl. Phys. 56, 1398 (1984).

[4] M. Geva, C. Cohen, O. Danziger, F. Dothan, L. Friedland, L. A. Levin, S. Maharshak, and J. L. Hirshfield, IEEE Trans. Plasma Sci. PS-15, 583 (1987).

[5] R. R. Prasad and M. Krishnan, Nucl. Instrum. Methods Phys. Res. B 26, 65 (1987).

[6] E. Del Bosco, S. W. Simpson, R. S. Dallaqua, and A. Montes, J. Phys. D 24, 2008 (1991).

[7] S. W. Simpson, R. S. Dallaqua, and E. Del Bosco, J. Phys. D 29, 1040 (1996).

[8] E. Del Bosco, R. S. Dallaqua, J. A. Bittencourt, and G. O. Ludwig, IEEE Trans. Plasma Sci. 17, 701 (1989).

[9] R. R. Prasad and M. Krishnan, J. Appl. Phys. 61, 4464 (1987).

[10] M. Geva, M. Krishnan, and J. L. Hirshfield, Nucl. Instrum. Methods Phys. Res. 186, 183 (1981).

[11] E. Del Bosco, R. S. Dallaqua, G. O. Ludwig, and J. A. Bittencourt, Appl. Phys. Lett. 50, 1716 (1987).

[12] R. S. Dallaqua, S. W. Simpson, and E. Del Bosco, IEEE Trans. Plasma Sci. 24, 539 (1996).

[13] Y. Yue and S. W. Simpson, J. Phys. D 29, 2866 (1996).

[14] R. S. Dallaqua, S. W. Simpson, and E. Del Bosco, J. Phys. D 30, 2585 (1997).
[15] R. W. Motley, Q Machines (Academic, New York, 1975).

[16] N. Rostoker and A. C. Kolb, Phys. Rev. 124, 965 (1961).

[17] R. J. Commission, C. A. Ekdahl, K. B. Freese, K. F. McKenna, and W. E. Quinn, Phys. Rev. Lett. 39, 137 (1977).

[18] W. A. Perkins and R. F. Post, Phys. Fluids 6, 1537 (1963).

[19] M. Petravic, L. G. Kuo, E. G. Murphy, and D. R. Sweetman, Phys. Fluids 7, 988 (1964).

[20] G. A. Hallock, E. B. Hooper, Jr., and J. H. Foote, Phys. Fluids 26, 314 (1983).

[21] F. F. Chen, Phys. Fluids 10, 1647 (1967).

[22] M. J. Hole and S. W. Simpson, Phys. Plasmas 4, 3493 (1997).

[23] M. J. Hole and S. W. Simpson, IEEE Trans. Plasma Sci. 27, 620 (1999).

[24] R. R. Prasad and M. Krishnan, J. Appl. Phys. 61, 113 (1987).

[25] M. J. Hole and S. W. Simpson, J. Phys. D 34, 3028 (2001).

[26] F. F. Chen, Phys. Fluids 8, 1323 (1965).

[27] F. F. Chen, Phys. Fluids 9, 965 (1966).

[28] M. J. Hole, Ph.D. thesis, University of Sydney, 2001.

[29] Lyman Spitzer, Jr., Physics of Fully Ionized Gases (Wiley, New York, 1962).

[30] S. W. Simpson and P. Kovitya, Appl. Phys. Lett. 43, 752 (1983).

[31] M. Mitchner and C. H. Kruger, Partially Ionized Gases (Wiley, New York, 1973).

[32] Handbook of Mathematical Functions, edited by M. Abramowitz and I. A. Stegun (Dover, New York, 1970). 
[33] M. N. Rosenbluth, N. A. Krall, and N. Rostoker, Nucl. Fusion Suppl. 1, 143 (1962).

[34] A. B. Mikhailovskii, Instabilities of an Inhomogeneous Plasma, Theory of Plasma Instabilities Vol. 2 (Consultants Bureau, New York, 1973).
[35] T. D. Rognlien, J. Appl. Phys. 44, 3505 (1973).

[36] T. K. Chu, B. Coppi, H. W. Hendel, and F. W. Perkins, Phys. Fluids 12, 203 (1969).

[37] F. F. Chen, Phys. Fluids 7, 949 (1964). 